\title{
Analytic description of the image to patient torso registration problem in image guided interventions
}

\author{
Vincenzo Ferrari ${ }^{1,2}$, Andrea Moglia ${ }^{2}$, Mauro Ferrari ${ }^{2,3}$ \\ 1. Department of Information Engineering, University of Pisa, Pisa, Italy. 2. EndoCAS Center, University of Pisa, Pisa, Italy. \\ 3. Vascular Surgery Unit, Cisanello Hospital, Pisa, Italy.
}

Correspondence: Vincenzo Ferrari, PhD. Address: Department of Information Engineering, University of Pisa, Pisa, Italy. Email: vincenzo.ferrari@unipi.it

Received: July 9, 2015

Accepted: July 28, $2015 \quad$ Online Published: August 6, 2015

DOI : $10.5430 /$ jbei.v1n1p35

URL: http://dx.doi.org/10.5430/jbei.v1n1p35

\begin{abstract}
Objective: The accurate registration of virtual pre-operative information of the human body anatomy, obtained as images with imaging devices, with real intra-operative information is one of the key aspects on which effective Image Guided Surgery (IGS) is based. The registration of pre-operative images on the real patient, during abdominal and thoracic interventions, is influenced by many parameters, which in many cases are influenced each other, thus making it often difficult to define the problem and consequently to solve it for each specific kind of intervention. The objective of this paper is to obtain an analytic description of the 3D image to patient registration problem, which can be more intuitive than the traditional textual descriptions.
\end{abstract}

Methods: The problem is formalized and various parameters affecting the registration are macro-classified in function of their nature.

Results: The problem is analytically described discussing for each macro-category of parameters potential solutions to avoid or to reduce their contribution to the registration error.

Conclusions: The availability of an analytic description of the image to patient torso registration problem can be beneficial for teaching IGS, to describe existing registration strategies, and to search new ones for each kind of surgery using a systematic approach.

\section{Key words}

Image guided surgery, Deformable registration, Surgical navigation, Abdominal and thoracic surgery

\section{I ntroduction}

Image to image registration is the process of determining the correspondence between two images, namely source and target image ${ }^{[1]}$. The determination of such a correspondence requires the geometrical or spatial mapping (or spatial transformation) of the source image so that it can be aligned with the target image. The mapping can be from one 3D coordinates system (source image) to another 3D coordinates system (target image) in case of computed tomography (CT) or magnetic resonance imaging (MRI), or from 2D to 2D in the case of ultrasound or C-Arm, and also in general a 2D image to a 3D image and vice-versa can be mapped. This work deals with 3D to 3D mapping, but almost all the considerations can be applied to the other cases. Transformations used for registration purposes can be subdivided into 
rigid and non-rigid ones. In the first case, the distance between all points in the image is preserved. Only translations and rotations occur from one coordinates system to another. This kind of transformation can be represented with homogeneous $4 \times 4$ transformation matrices for 3D to 3D mapping. In sharp contrast, in non-rigid transformations the distance between all points varies, so that lines are mapped to curves ${ }^{[1]}$ and also the transformations can be static or dynamic, as in the case of four dimensional computed tomography (4D CT) where the images move as the anatomy due to physiological movements.

In image to patient registration the goal is to determine the correspondence between pixel (in the 2D case) or voxel (in the 3D case) of the image with the corresponding point of the human body.

The accurate registration of virtual pre-operative information of the human body anatomy, obtained as images after radiological acquisitions, with real intra-operative information, acquired during surgical interventions on the real patient, is one of the key aspects on which effective computer assisted surgery (CAS) and in particularly image guided surgery (IGS) is based ${ }^{[2]}$. In particular, the registration of virtual pre-operative information with real intra-operative information (patient and surgical instruments) allows the surgeon to employ augmented-reality systems to see covered structures before reaching them (see Figure 1) ${ }^{[3-7]}$, surgical navigators to see the scene (anatomy and instruments) from different point of views ${ }^{[8,9]}$ and smart mechatronics devices which, knowing the anatomy, assist him/her in an active way ${ }^{[10,11]}$. All these aspects are potentially useful in terms of safety and efficiency for the patient, the physicians, and the health system as well.

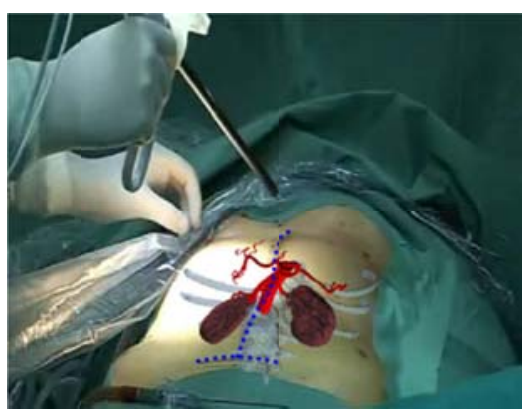

Figure 1. An augmented reality system at work in the operative room
In order to develop similar systems it should be noticed that pre-operative information is generally acquired in the reference frame of the radiological instrumentation just before or days before the surgical procedure, whereas the intraoperative information is related to the reference frame of the surgical room (generally defined by means of a tracking system) during the intervention.

Several image to image registration approaches based on general purpose methods of rigid and non-rigid registration have been proposed ${ }^{[12,13]}$. Moreover, many techniques of computational intelligence have been developed ${ }^{[14]}$. These solve the general problem of registration between two images compensating for different positioning of the patient, device scanner aberration, and in some cases anatomy shifts ${ }^{[15]}$, obtaining an image fusion between the source and the target image.

Image to patient rigid registration is used in many CAS and IGS applications, and various analytic and experimental accuracy evaluation works have been performed and reported. The application of rigid registration is obviously limited to bones and head because of the not-rigid nature of the abdominal and torso structures, and soft tissues in general.

Non-rigid registration algorithms produce good results for images fusion, in which the input and the target are both images (for example CT to MRI) ${ }^{[16]}$. Their application to IGS would require dense and volumetric intra-operative information of the patient's anatomy placed on the surgical table, e.g. using intra-operative CT, MRI, 3D RA (Rotational Angiography) or 3D Ultrasound. In general, using intra-operative imaging, the registration of the soft tissues can be avoided or simplified ${ }^{[17]}$. A list of applications that do not require intra-operative registration, as the information is intrinsically coherent to the patient, has been reported by Hawkes et al. ${ }^{[18]}$. The use of intra-operative imaging is often very precise in terms of voxel localization in space and also in time, thanks to the availability of 4D scanners, while often the visual image quality offered by intra-operative radiological scanners is lower than the visual image quality offered by traditional ones. An additional issue is represented by the low availability of volumetric imaging devices in the operating rooms, which could be overcome in the early future at least for some kinds of devices. 
Some authors proposed to follow the movements of internal organs elaborating endoscopic images: applying artificial markers directly on organs surfaces ${ }^{[19]}$, while recent research works on machine vision proposed to avoid the need of artificial markers performing a video tracking of the organs exposed surfaces ${ }^{[20,21]}$. At present only in-vitro and animal tests have been performed using the previous techniques, since there are obvious troubles related to the insertion of artificial markers on organs parenchyma, and the presence of fat tissue on organ surface seriously compromise the clinical application of such techniques based on the reconstruction of the organs (not exposed) surfaces.

The trajectory of target points, contained in the target volume of interest for each specific intervention, can be predicted during the breathing at given respiratory states ${ }^{[22]}$. Recent studies have proposed dynamic models for reproducing the entire trajectory of target points during the respiratory cycle ${ }^{[23,24]}$. However, they can fail because of the displacements generated by other physiological effects (not contemplated in the models) or from a not correct re-positioning of the patient (in the operative room in respect to the radiological department). In any case this approach could offer good results in the future for the replication of movements and deformations of abdominal and torso structures in image-guided surgical treatments, following a methodological approach to the problem, which takes into account all sources of error and not only breathing.

In this regard, a thorough biomechanical model of the anatomy has been proposed, theorized, supported and promoted by the European Union ${ }^{[25-27]}$ in order to provide a complete dynamic registration compensating all physiological parameters and decubitus of the patient in the surgical room, which may differ from those in the radiological department.

All non-rigid techniques, static or dynamic require a preliminary coarse registration as starting point for more accurate deformable (non-rigid) approaches ${ }^{[13,28]}$. Today, rigid body registration is the only approach used for this initial alignment thanks to the amount of published works which evaluated its mathematical properties and to the existence of mathematically optimal solutions to the problem ${ }^{[29,30]}$. The method used for this initial registration is fundamental in order to reduce the starting registration error, and also it is important to know the error obtained because, in general, the non rigid registration algorithms are iterative and provide optimal local solutions which depend on the starting conditions. Even though there are some studies on the accuracy of rigid body registration for cranial and extra cranial targets, there are no specific works for the estimation of errors in the abdomen and torso ${ }^{[31]}$.

The registration of pre-operative 3D images on the real patient, during abdominal and thoracic interventions, is influenced by many parameters, which in many cases are influenced each other, thus making often difficult to define the problem and consequently to solve it for each specific kind of intervention with a methodological approach. The problem is well known as described in many medical and bioengineering papers. The objective of this paper is to obtain an analytic description of the 3D image to patient registration problem, which can be more intuitive, at least for technicians, than the traditional textual descriptions mainly published by radiotherapists ${ }^{[32]}$.

\section{Methods}

In the next paragraphs the problem is formalized and the various parameters affecting the registration are macro-classified in function of their nature in order to obtain an analytic description of the 3D images to patient torso registration problem in image guided interventions.

\subsection{The abdomen registration problem for I GS}

The sources of the problem are the position of the center of mass of each voxel $i$ in the reference frame of the radiological device $r d$ that we define $V r d_{i}$. The targets are the corresponding target moving points in the patient's anatomy $\operatorname{Vsr}_{i}(t)$, which can be referred to a steady reference frame in the surgical room as the reference frame of a tracker or a frame attached to the patient. From a surgical point of view, it would be ideal to know with extreme precision where each $\operatorname{Vsr}_{i}(t)$, corresponding to a specific $V_{r d}$, is at each instant as depicted in Figure 2. 


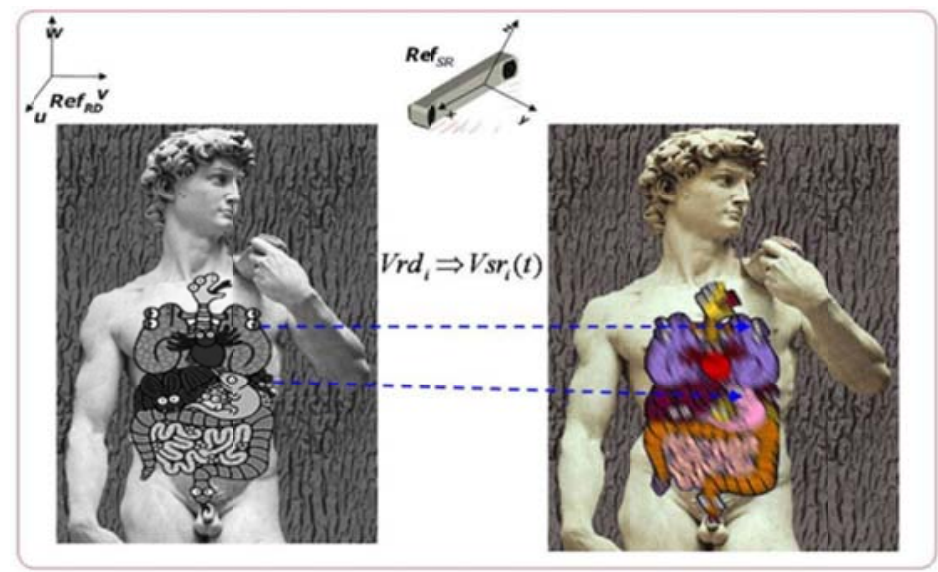

Figure 2. The voxel barycentre $V r d_{i}$ (left), statically acquired in the radiological device $(r d)$ reference frame has to be mapped on the moving corresponding point $\operatorname{Vsr}_{i}(t)$ (right), in the surgical room (sr) reference frame.

Analytically, the problem is to estimate for each voxel barycentre $V r d_{i}$ the corresponding instantaneous position in the patient in a surgical room steady reference frame $\hat{V} s r_{i}(t)$ that guarantees:

$$
\hat{V} s r_{i}(t) \cong V s r_{i}(t)
$$

Radiotherapists experience a similar problem every day ${ }^{[32]}$. However, they need to localize only a restricted target area and not an entire anatomical district, as in general for IGS (surgical navigation, augmented-reality, etc...). Additionally, the reference systems considered by radiotherapists are all those of the planning room, normally a CT with a positioning system based on laser crosses, which are mechanically calibrated with all reference systems in the bunker room (normally a proton accelerator, a positioning system based on laser crosses, and in case a 2D imaging device). In that case they do not have to register the patient, but to reposition him/her in order to align laser crosses with reference markers on the skin of the patients, because all reference systems are calibrated together.

\subsection{Parameters affecting the registration}

The process of abdominal images registration is affected by the following parameters.

In the first instance, any orientation changes in the position of bed/operating table on which the patient lies (e.g., Trendelenburg, anti-Trendelenburg, lateral tilt and other typical positioning of the patient) will induce a gravitational displacement of mobile internal organs. Second, alteration in the configuration of the patient support has to be taken into account as this may introduce a deformation: in particular segmented beds when tilted in various directions impose deformations/displacement of the abdominal cavity and its contents. The same is true also for concave beds (commonly used with CT and MRI devices).

The lower part of the abdominal cavity is delimited by the pelvic basin (rigid) and upper part by the thoracic cage (semi-rigid); hence, the relative positioning of these anatomical structures, depending by the patient decubitus, influences the location of the contained anatomies. Patient decubitus affects also the position of the limbs, which can impact on the location of the intra-abdominal structures, since their movement can deform the torso.

The radiological devices used and all post-processing phases performed, e.g. segmentation, introduce errors. In particular the use of specific radiological devices or specific surgical techniques imposes some requirements on the patient that, in turn, may introduce errors in the localization of targets. For example, during some abdominal interventions, an arm of the patient may lie along the trunk. Thus, it would be advantageous to acquire the patient in this position, but CT scanning of 
arms, close to the abdomen, introduces artifacts (scattering). For this reason during CT scanning the arms are (generally) positioned underneath the head, thus changing the patient setup. Furthermore, scanning with CT machines generally requires breath holding of the patient in inspiration during the acquisition of the images dataset to ensure a steady volume. However in deep inhalation there is a descent of the diaphragm and of those organs in contact with it. This effect is used routinely by radiologists during ultrasound scanning for improved exposure and examination of organs. They know that abdominal organs can be moved up to $6 \mathrm{~cm}-8 \mathrm{~cm}$ in maximum inhalation. This effect has also been measured by us in a 65 years old female patient, aligning her spine on a CT dataset acquired during normal breathing to a CT dataset acquired during a period of inhalation apnea. The result showed in Figure 3 demonstrates that the kidneys have been moved up by $27 \mathrm{~mm}$, and the displacement of the spleen is $40 \mathrm{~mm}$.

Figure 3. The spine of a patient, acquired during normal breathing, is aligned to a dataset (segmented and coloured) acquired during inhalation apnea. The displacement of spleen and kidneys can be evaluated.

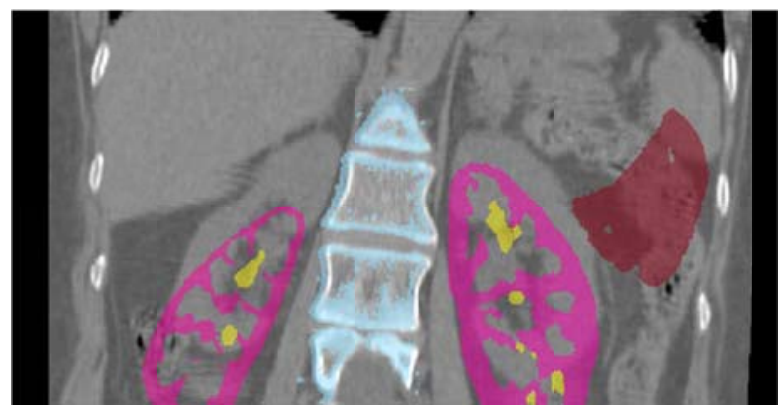

Normal breathing introduces a displacement and a deformation on the lungs and to the upper abdominal organs. The diaphragm has a maximum excursion during normal breathing of about $\pm 7 \mathrm{~mm}$ around its median position ${ }^{[33]}$. As a consequence, the structures in direct contact with the diaphragm will be displaced by a similar excursion and sustain some deformation. While other experiments reported a maximum excursion of $\pm 7.5 \mathrm{~mm}$ for the abdominal wall, on the skin, during normal breathing ${ }^{[34]}$. The other internal organs, passive elements enclosed between the diaphragm and the abdominal wall, would not move (probably) more than them.

An important type of movements is generated by the gastrointestinal tract and is related to the state of filling of the stomach and contractile movement of the intestine (caused also by the anaesthesia); all of these movements induce displacement of adjacent structures and thus generate errors that are particularly relevant to intra-peritoneal structures. An example is depicted in Figure 4.

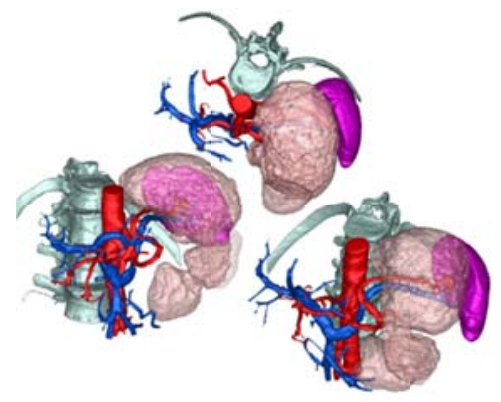

Figure 4. 3D model reconstructed from real data acquired from a patient with the stomach full of water. The stomach (pink-transparent) touches the spleen (purple) and lies on splenic vessels. If the stomach were empty, the position of the spleen would be more inward and upward.
Other changes in physiological parameters can move the anatomies. The heart beating poses troubles not only in cardio surgery but also in vascular surgery, where the rhythmic changes of the blood pressure introduces visible deformations on the arterial walls. Furthermore the effect of the insufflations in laparoscopy can be considered a variation in the abdominal pressure. Its effect on the abdominal wall was documented by Song et al. ${ }^{[35]}$. In general all physiological parameters (Young-Modulus of each structures, blood pressure, abdominal pressure, muscles contractions, position and velocity of elements, etc...) can be a source of movement which should be taken into account.

Another class of dissimilarities appears when the surgeon modifies the topology of the anatomy during the intervention. In this case the pre-operative information may not be topologically consistent with the real anatomy and consequently the virtual model should be adjusted. 


\subsection{Analytic description of the problem}

In this paragraph the problem defined in 2.1 is described in more details in function of the parameters affecting the registration described in 2.2 .

The position of each point in the patient is determined by the following macro-categories of parameters:

- $\quad b c(t)$ is the bed configuration in terms of angles relative to the floor, shape and covering material at time $t$;

- $\operatorname{dec}(t)$ is the decubitus of the patient on the bed at time $t$;

- $p p(t)$ indicate all physiological parameters at time $t$.

So equation (1) becomes:

$$
\hat{V s r}_{i}(t) \cong V s r_{i}(b c(t), \operatorname{dec}(t), p p(t))
$$

3D radiological devices acquire "frozen" voxels at particular instants and often some delay occurs between the acquisition of the various voxels of an image. Generally, a generic voxel $i$ is acquired at instant $t_{i}$ and depends on $b c\left(t_{i}\right), \operatorname{dec}\left(t_{i}\right)$, and $p p\left(t_{i}\right)$ of the patient at the acquisition time. Neglecting the artifacts introduced by the radiological device, $\operatorname{Vrd}_{i}$ can be defined as:

$$
\operatorname{Vrd}_{i} \equiv \operatorname{Vrd}_{i}\left(b p\left(t_{i}\right), \operatorname{dec}\left(t_{i}\right), p p\left(t_{i}\right)\right)
$$

$\hat{V}_{s} r_{i}(t)$ can be determined as function of the pre-operative information expressed by all $\operatorname{Vrd}{ }_{i}$, the time, and in case by other source of information relative to surgical and diagnostic devices (like endoscopic cameras) and the patient (like position sensors, physiological sensors, etc...) that satisfy equation (2):

$$
\forall \bar{t}, i \quad{\hat{V} s r_{i}}_{i}(\bar{t})=F^{\prime}\left(\operatorname{Vrd} d_{1} \ldots V r d_{n}, \ldots, \bar{t}\right) \mid \hat{V} s r_{i}(\bar{t}) \cong V s r_{i}(b p(\bar{t}), \operatorname{dec}(\bar{t}), p p(\bar{t}))
$$

Where $F^{\prime}\left(V r d_{i} \ldots V r d_{n}, \ldots, t\right)$ means a function of all the available $V r d_{i}$ and possible additional parameters relative to the devices, the patient and the time.

From (4), a weaker condition can be written as following:

$$
\forall \bar{t}, i, j \quad\left\|\hat{V s} r_{i}(\bar{t})-\hat{V s} r_{j}(\bar{t})\right\| \cong\left\|V s r_{i}(\bar{t})-V s r_{j}(\bar{t})\right\|
$$

Relation (5) implies that the configurations between real points, expressed by $V s r_{i}(\bar{t})$ and estimated points $\hat{V s} r_{i}(\bar{t})$ have to be as similar as possible, in terms of distances between each other. Relation (5) remains valid applying rigid transformations on all points. As a consequence, the problem can be decomposed as follows: a rigid transformation for the alignment of the two reference frames ( $r d$ and $s r$ ), followed by a deformation of the preoperative virtual information to the real information adjusting the relative distances of the points, at each instant. Since this deformation is in general time depending, and deformable registration is often used in IGS for static (not time depending) deformable registrations, in this paper we prefer to define it as configuration adaptation. 
The same approach is used in every non-rigid method for the registration of static medical images (coarse followed by fine registration or rigid followed by non rigid registration) ${ }^{[28,36]}$. In our case the problem is a bit more complicated because a static points cloud, corresponding to the pre-operative information ( $V r d_{i}$ points), has to be aligned with a "moving" points cloud corresponding to the intra-operative information (moving $V s r_{i}(t)$ points), but the approach can be the same.

The order of the two operations, rigid transformation and configuration adaptation, could be swapped, but the classic order, rigid followed by non rigid registration, has to be preferred on the ground of some intuitive considerations. Obviously it is not possible to describe points trajectories $\operatorname{Vsr}_{i}(t)$ using only the static values of all $\operatorname{Vrd}_{i}$. Further intra-operative information is required. A large number of intra-operative information could be acquired using additional radiological volumetric and potentially 4D devices in the surgical room. This information would describe the trajectories of many points of the patient body during the intervention. The trajectories of the not acquired points by the intra-operative radiological device could be estimated using, for example, interpolation. Unfortunately almost all surgical rooms have no volumetric radiological devices (like open MRI or similar). However, the acquisition of some intra-operative data, as those acquired by means of tracking system or by means of ultrasound on or inside the body, can offer important conditions for biomechanical/physiological models similar to those documented by Blackall and McClelland demonstrating the possibility to predict points position during breathing ${ }^{[23,24]}$. By measuring only a part of the intra-operative information, other information could be estimated. This estimation could be enhanced during the intervention, depending on the precision and on the type of the available intra-operative devices. For example when the surgeon discovers new distinguishable anatomical landmarks, he/she can acquire this point by means of localized instruments and cameras. This information could offer the position of some $V s r_{i}$, which could be employed as a useful new condition for biomechanical or motion models. All this information will be represented in a reference frame of the surgical room (fixed with a tracker), so a preliminary change of reference system on the pre-operative data is functional in order to use coherent information in the biomechanical or motion models. This is a reason to enforce the traditional order of the transformations: rigid followed by non rigid registration one.

The rigid transformation can be performed by aligning either fiducial points or fiducial surfaces on the patient, acquired in the pre-operative radiological device reference frame $r d$ and in the surgical room reference frame $s r$, assuming the patient, or a part of the patient, as a rigid object ${ }^{[29,30]}$. Deformations of the fiducials structure composed by elements, such as points of a cloud or points characterizing a surface, introduce systematic errors in the registration. In order to minimize the registration error, at least on fiducials elements, each fiducial point (or fiducial surface) in the proximity of steady element on the patient has to be chosen, and its configuration has to be as replicable as possible ${ }^{[37]}$. In this case, a rigid registration can be performed at the beginning of the intervention and should be repeated only if the bed configuration $b c$, or the patient decubitus dec, will be changed.

A rigid transformation on $\mathrm{Vrd}_{i}$ can be written, using Cartesian coordinates, in terms of rotation matrix $R$ and translation vector $T$, as follows:

$$
\tilde{V} s r_{i}\left(b p\left(t_{i}\right), \operatorname{dec}\left(t_{i}\right), p p\left(t_{i}\right)\right)=R \cdot V r d_{i}\left(b p\left(t_{i}\right), \operatorname{dec}\left(t_{i}\right), p p\left(t_{i}\right)\right)+T
$$

Then, the configuration adaptation becomes:

$$
\forall \bar{t}, i \quad{\hat{V} s r_{i}}_{i}(\bar{t})=F\left(\tilde{V} s r_{1} \ldots \tilde{V} s r_{n}, \ldots, \bar{t}\right)
$$

which has to satisfy (5). Function $F$ will be very similar to $F^{\prime}$, the only difference is that $F$ works with input and output data in the $s r$ reference. $F$ will depend on the rigidly registered pre-operative voxels barycentres $\tilde{V}_{s}\left(b p\left(t_{i}\right), \operatorname{dec}\left(t_{i}\right), p p\left(t_{i}\right)\right)$ and other parameters relative to the patient (like position sensors, physiological sensors, etc...) and time. 


\section{Results}

The general problem can be summarized with the following steps:

1) To determine the change of reference system (eq. 6) to rigidly move each voxel barycentre $V r d_{i}$, expressed in the radiological device reference frame $r d$, in the corresponding $\tilde{V}_{s} r_{i}$ expressed in a steady reference system $s r$ in the surgical room;

2) To define a configuration adaptation function $F$ (eq. 7) and its required parameters able to estimate anatomy deformations (eq. 5) in order to move each pre operative acquired and rigidly registered $\tilde{V}_{s} r_{i}$ on the corresponding $\operatorname{Vsr}_{i}(t)$ as required by equation (4), which become:

$$
\forall \bar{t}, i \quad \hat{V} s r_{i}(\bar{t})=F\left(\tilde{V} s r_{1}\left(b p\left(t_{i}\right), \operatorname{dec}\left(t_{i}\right), p p\left(t_{i}\right)\right) \ldots \tilde{V} s r_{n}\left(b p\left(t_{i}\right), \operatorname{dec}\left(t_{i}\right), p p\left(t_{i}\right)\right), \ldots, \bar{t}\right) \mid \hat{V} s r_{i}(\bar{t}) \cong V s r_{i}(b p(\bar{t}), \operatorname{dec}(\bar{t}), p p(\bar{t}))(8)
$$

\section{Discussion}

The goal of this paper is not to find a solution for the registration of each anatomical structure of the human torso for each kind of intervention, but simply to propose the 3D image to patient registration problem from an analytic point of view.

One of the author experienced as a teacher that this analytic description is useful to allow students to understand the problem from a general point of view and then to analyze possible solution for each specific case.

This description can be also useful to describe existing registration strategies and to search new ones for each kind of surgery using a systematic approach.

At first, one has to note that also in case of rigid structures inside the torso, e.g. a vertebra, the registration is in general simpler than on a deformable tissue, but not trivial. The distance between points inside a vertebra remains approximately constant, but the pose of a vertebra is influenced by the $b p$, dec and $p p$ factors: the shape of the bed influences the spine and vertebras configuration, which is also influenced by the patients' decubitus, and finally phisiological parameters like breathing can move the spine. For this reason in spine IGS, to obtain high registration accuracy, a frame is rigidly fixed directly on the vertebra and a rigid registration (e.g. using $\mathrm{x}$-Ray projections) is performed ${ }^{[38,39]}$ to determine the transformation (as eq. 6) between the radiological device reference frame $r d$ and the reference system of the frame attached to the spine that is, following the notation introduced in this paper, the $s r$ reference frame. This rigid registration is enough to align each $\tilde{V}_{s} r_{i}$ on the corresponding $V s r_{i}$ and the configuration adaptation function $F$ reported in equation (7) and (8) is not required since the virtual pre-operative vertebra and the real vertebra are rigid and so $b c$, dec and $p p$ parameters do not affect vertebra points positions in the $s r$ reference frame attached to the vertebra. If is not possible to employ a steady reference frame fixed with the anatomy, the registration of rigid structures is prone to large errors as for deformable tissues.

In the general case of soft tissues, or rigid structures without a steady reference frame, one can employ procedures routinely used by radiotherapists to meticulously reproduce the patient settings during the treatment as in the planning room. By following radiotherapists work, bed positioning and its shape during the acquisition of medical datasets has to be chosen accordingly to the bed configuration used inside the surgical room for the specific intervention (considering the requirements of the radiological device and the type of intervention to be performed). In this case $b c$ in the surgical room will be similar, as more as possible, to the one during the acquisition of the pre-operative image. Furthermore, during the intervention the exact decubitus of the patient has to be replicated as during the radiological scanning to obtain the same relative position of the basin and the thoracic cage, in order to obtain a dec in the surgical room similar, as more as 
possible, to the one during the acquisition of the pre-operative image. A realignment of these structures needs immobilizeation devices and/or additional iterative work in the surgical room in order to find a perfect correspondence between pre-operative and intra-operative patient decubitus ${ }^{[40]}$. Furthermore, the position of arts affects the arrangement of some abdominal organs, in particular: arms position influences upper abdominal structures, while legs position influences the lower ones. For this reason, radiotherapists immobilize arms or legs depending on the body part they have to treat in order to obtain a patient decubitus in the treatment room like in the planning room. The steps to follow in case of image guided interventions are the same. The realignment procedure allows to simplify the configuration adaptation function $F$ (eq. 8) removing the need to simulate the changes in $\tilde{V}_{s} r_{i}$ in respect to $V s r_{i}$ due to $b c$ and $d e c$ parameters.

If there are the minor differences between for $\tilde{V} s r_{i}$ and $V s r_{i}$ due to $b c$ and $\operatorname{dec}$ factors, respiratory motion models that correlate target points trajectories, pre operatively acquired with radiological scanners, with the respiratory cycle, acquired with breathing monitors ${ }^{[41-45]}$ allow to precisely implement configuration adaptation function $F$. A review of respiratory motion models is in Ref. ${ }^{[46]}$. They can fail in a real scenario because of the displacements generated by other physiological effects (not contemplated in the motion models) or from a not correct re-positioning of the patient on the surgical bed. In case of differences in $b c$ or $d e c$ parameters a predictive model cannot be accurate since the basal position of each $\tilde{V}_{s} r_{i}$ (at a defined instant of the breathing cycle e.g. at the end of the exhalation phase) is different to the corresponding $V s r_{i}$.

The using of intra-operative device like CT or MRI simplifies the problem but their availability, for logistic and economic reasons, is limited. In any case, more simple and cheap intra-operative imaging devices like 3D ultrasound or 3D RA, which will be widespread in the near future thanks to the lowering of their price as portable unit, allows to avoid the change of reference frame for each patient (eq. 6). It is possible since the scanner positioned in the operating room can be easily and precisely calibrated with the surgical room reference frame $s r$. Furthermore the acquisition of the anatomy directly on the surgical bed allows to dramatically simplify the problem, by removing differences between $\tilde{V}_{s} r_{i}$ and $V s r_{i}$, due to $b c$ and dec factors. Intra-operative scanners could be also useful in all cases where the use of pre-operative images cannot be avoided due to the type and the quality of the information required performing the intervention. As an example 3D RA are able to acquire fine morphologic information on bones (orthopaedics) and on arteries (cardio vascular surgery) but the quality and the quantity of the information, at least for some anatomical structures, is not comparable with CT and MRI. In these cases intra-operating imaging devices like 3D RA offers the possibility to precisely perform the change of reference frame (eq. 6) using not just few points, but using an entire volume of information. The same approach can be performed with other kind of intra-operative imaging device e.g. an ultrasound to acquire clearly distinguishable internal anatomical references ${ }^{[47]}$ or directly the entire shape of the structure of interest ${ }^{[48]}$.

In case of changes due to $b c$ and $d e c$ factors, the availability of dense intra-operative information allows, theoretically, to proceed for its compensation by means of the implementation of complex deformable algorithms having a lot of information on the target configuration.

As analyzed there are different possibilities to try to solve the registration problem. The open question is to understand what the best approach for each anatomical structure and for each kind of intervention is.

\section{Conclusion}

The registration of pre-operative 3D images on the real patient, during abdominal and thoracic interventions, is influenced by many parameters, which in many cases are influenced each other.

The availability of an analytic description of the problem can be beneficial for teaching IGS and to describe existing registration strategies and to search new ones for each kind of surgery using a systematic approach. 
The result of the analysis highlights the complexity of the problem, the need to simplify it using a methodological approach, and the usefulness of intra-operative imaging devices, like 3D RA or 3D ultrasound, which will be widespread in the near future thanks to the lowering of their price as portable units.

\section{References}

[1] Holden M. A Review of Geometric Transformations for Nonrigid Body Registration. Medical Imaging, IEEE Transactions on. 2008; 27(1): 111-28. http://dx.doi.org/10.1109/TMI.2007.904691

[2] Robb RA, Director BIR. Biomedical imaging: past, present and predictions. Journal of Medical Imaging Technology. 2006; 1: 25-37.

[3] Nicolau S, Soler L, Mutter D, et al. Augmented reality in laparoscopic surgical oncology. Surg Oncol. 2011; 20(3): 189-201. Epub 2011/08/02. PMid: 21802281.

[4] Berryman DR. Augmented reality: a review. Medical reference services quarterly. 2012; 31(2): 212-8. http://dx.doi.org/10.1080/02763869.2012.670604

[5] Ferrari V, Megali G, Troia E, et al. A 3-D mixed-reality system for stereoscopic visualization of medical dataset. IEEE Trans Biomed Eng. 2009; 56(11): 2627-33. PMid: 19651551. http://dx.doi.org/10.1109/TBME.2009.2028013

[6] Kersten-Oertel M, Jannin P, Collins DL. The state of the art of visualization in mixed reality image guided surgery. Computerized medical imaging and graphics: the official journal of the Computerized Medical Imaging Society. 2013; 37(2): 98-112. PMid: 23490236. http://dx.doi.org/10.1016/j.compmedimag.2013.01.009

[7] Pietrabissa A, Morelli L, Ferrari M, et al. Mixed reality for robotic treatment of a splenic artery aneurysm. Surgical endoscopy. 2010; 24(5): 1204. PMid: 19826869. http://dx.doi.org/10.1007/s00464-009-0703-0

[8] Mezger U, Jendrewski C, Bartels M. Navigation in surgery. Langenbeck's archives of surgery/Deutsche Gesellschaft fur Chirurgie. 2013; 398(4): 501-14. http://dx.doi.org/10.1007/s00423-013-1059-4

[9] Megali G, Ferrari V, Freschi C, et al. EndoCAS navigator platform: a common platform for computer and robotic assistance in minimally invasive surgery. Int J Med Robot. 2008; 4(3): 242-51. PMid: 18698670. http://dx.doi.org/10.1002/rcs.203

[10] Freschi C, Troia E, Ferrari V, et al. Ultrasound guided robotic biopsy using augmented reality and human-robot cooperative control. Conf Proc IEEE Eng Med Biol Soc. 2009; 2009: 5110-3. PMid: 19963882. http://dx.doi.org/10.1109/IEMBS.2009.5332720

[11] Lang JE, Mannava S, Floyd AJ, et al. Robotic systems in orthopaedic surgery. The Journal of bone and joint surgery British volume. 2011; 93(10): 1296-9. Epub 2011/10/05. PMid: 21969424. http://dx.doi.org/10.1302/0301-620X.93B10.27418

[12] Zitova B, Flusser J. Image registration methods: a survey. Image and vision computing. 2003; 21(11): 977-1000. http://dx.doi.org/10.1016/S0262-8856(03)00137-9

[13] Maintz JA, Viergever MA. A survey of medical image registration. Medical image analysis. 1998; 2(1): 1-36. http://dx.doi.org/10.1016/S1361-8415(01)80026-8

[14] Ramirez L, Durdle N, Raso V, editors. Medical image registration in computational intelligence framework: a review. Electrical and Computer Engineering, 2003 IEEE CCECE 2003 Canadian Conference on; 2003: IEEE. http://dx.doi.org/10.1109/ccece.2003.1226069

[15] Miga M, Paulsen KD, Lemery JM, et al., editors. Model-updated image guidance: initial clinical experiences with gravity-induced brain deformation. Biomedical Imaging, 2002 5th IEEE EMBS International Summer School on; 2002: IEEE.

[16] Commowick O, Arsigny V, Isambert A, et al. An efficient locally affine framework for the smooth registration of anatomical structures. Med Image Anal. 2008; 12(4): 427-41. Epub 2008/03/08. PMid: 18325825. http://dx.doi.org/S1361-8415(08)00008-X [pii]10.1016/j.media.2008.01.002.

[17] Feuerstein M, Mussack T, Heining SM, et al. Registration-free Laparoscope Superimposition for Intra-Operative Planning of Liver Resection. 3rd Russian-Bavarian Conference on Biomedical Engineering; July 2007. p. 88-92.

[18] Hawkes DJ, Barratt D, Blackall JM, et al. Computational Models In Image Guided Interventions. Engineering in Medicine and Biology Society, 2005 IEEE-EMBS 2005 27th Annual International Conference; 2005. p. 7246-9. http://dx.doi.org/10.1109/iembs.2005.1616183

[19] Teber D, Guven S, Simpfendorfer T, et al. Augmented reality: a new tool to improve surgical accuracy during laparoscopic partial nephrectomy? Preliminary in vitro and in vivo results. Eur Urol. 2009; 56(2): 332-8. Epub 2009/05/30. PMid: 19477580. http://dx.doi.org/S0302-2838(09)00521-1 [pii] 10.1016/j.eururo.2009.05.017 
[20] Stoyanov D, Scarzanella MV, Pratt P, et al. Real-time stereo reconstruction in robotically assisted minimally invasive surgery. Med Image Comput Comput Assist Interv. 2010; 13(Pt 1): 275-82. Epub 2010/10/01. PMid: 20879241. http://dx.doi.org/10.1007/978-3-642-15705-9_34

[21] Haouchine N, Dequidt J, Peterlik I, et al., editors. Image-guided Simulation of Heterogeneous Tissue Deformation For Augmented Reality during Hepatic Surgery. ISMAR - IEEE International Symposium on Mixed and Augmented Reality 2013; 2013 2013-10-01 2013-10-04INFO:INFO_GR H.: Information Systems/H.5: INFORMATION INTERFACES AND PRESENTATION (e.g., HCI)/H.5.1: Multimedia Information Systems/H.5.1.1: Artificial, augmented, and virtual realities, I.: Computing Methodologies/I.3: COMPUTER GRAPHICS/I.3.5: Computational Geometry and Object Modeling/I.3.5.7: hysically based modeling international Adelaide Australia. http://dx.doi.org/10.1109/ismar.2013.6671780

[22] Olbricha B, Traubb J, Wiesnerb S, et al. Respiratory Motion Analysis: Towards Gated Augmentation of the Liver. CARS 2005 Computer Assisted Radiology and Surgery, 19st International Congress and Exhibition; 2005.

[23] Blackall JM, Ahmad S, Miquel ME, et al. MRI-based measurements of respiratory motion variability and assessment of imaging strategies for radiotherapy planning. Phys Med Biol. 2006; 51(17): 4147-69. PMid:16912374. http://dx.doi.org/10.1088/0031-9155/51/17/003

[24] McClelland JR, Blackall JM, Tarte S, et al. A continuous 4D motion model from multiple respiratory cycles for use in lung radiotherapy. Med Phys. 2006; 33(9): 3348-58. Epub 2006/10/07. PMid:17022231. http://dx.doi.org/10.1118/1.2222079

[25] Delingette H, Pennec X, Soler L, et al. Computational Models for Image-Guided Robot-Assisted and Simulated Medical Interventions. Proceedings of the IEEE. 2006; 94(9): 1678-88. http://dx.doi.org/10.1109/JPROC.2006.880718

[26] Riboldi M, Baroni G, Spadea M, et al. Implementation of FEM simulation techniques for organ motion modeling in prostate cancer radiotherapy. Radiotheraphy Oncology. 2006.

[27] Ayache N, Boissel J-P, Brunak S, et al. Towards Virtual Physiological Human: Multilevel Modelling and Simulation of the Human Anatomy and Physiology. 2006.

[28] Scheuering M, Rezk-Salama C, Barfufl H, et al. Augmented reality based on fast deformable 2D-3D registration for image-guided surgery. Proc SPIE Vol 4681, p 436-445, Medical Imaging 2002: Visualization, Image-Guided Procedures, and Display, Seong K Mun; Ed; May 2002. p. 436-45. http://dx.doi.org/10.1117/12.466948

[29] Arun KS, Huang TS, Blostein SD. Least-squares fitting of two 3-D point sets. IEEE Trans Pattern Anal Mach Intell. 1987; 9(5): 698-700. PMid:21869429. http://dx.doi.org/10.1109/TPAMI.1987.4767965

[30] Besl PJ, McKay ND. A Method for Registration of 3-D Shapes. IEEE Trans Pattern Anal Mach Intell. 1992; 14(2): 239-56. http://dx.doi.org/10.1109/34.121791

[31] Baroni G, Garibaldi C, Riboldi M, et al. 3D optoelectronic analysis of interfractional patient setup variability in frameless extracranial stereotactic radiotherapy. Int J Radiat Oncol Biol Phys. 2006; 64(2): 635-42. PMid:16198068. http://dx.doi.org/10.1016/j.ijrobp.2005.07.964

[32] van Herk M. Errors and margins in radiotherapy. Semin Radiat Oncol. 2004; 14(1): 52-64. Epub 2004/01/31. PMid: 14752733. http://dx.doi.org/S1053-4296(03)00084-5 [pii] 10.1053/j.semradonc.2003.10.003

[33] Ayoub J, Cohendy R, Dauzat M, et al. Non-invasive quantification of diaphragm kinetics using m-mode sonography. Can J Anaesth. 1997; 44(7): 739-44. PMid:9232305. http://dx.doi.org/10.1007/BF03013389

[34] Ferrari V, Megali G, Cappelli C, et al. Improving daily clinical practice with 3D patient-specific anatomical models: limits, methodologies and our experience. 6th International Conference on the Management of Healthcare \& Medical Technology HCTM; 3-5 October 2007.

[35] Song C, Alijani A, Frank T, et al. Mechanical properties of the human abdominal wall measured in vivo during insufflation for laparoscopic surgery. Surg Endosc. 2006; 20(6): 987-90. PMid:16738998. http://dx.doi.org/10.1007/s00464-005-0676-6

[36] Maintz JB, Viergever MA. A survey of medical image registration. Med Image Anal. 1998; 2(1): 1-36. Epub 2000/01/19. PMid: 10638851. http://dx.doi.org/S1361-8415(01)80026-8 [pii]

[37] Megali G, Ferrari V, Freschi C, et al. EndoCAS navigator platform: a common platform for computer and robotic assistance in minimally invasive surgery. The International Journal of Medical Robotics and Computer Assisted Surgery. 2008 ; 4 : 242-51. PMid:18698670. http://dx.doi.org/10.1002/rcs.203

[38] Shoham M, Burman M, Zehavi E, et al. Bone-mounted miniature robot for surgical procedures: Concept and clinical applications. Robotics and Automation, IEEE Transactions on. 2003; 19(5): 893-901. http://dx.doi.org/10.1109/TRA.2003.817075

[39] Kim H-J, Lee SH, Chang B-S, et al. Monitoring the Quality of Robot-Assisted Pedicle Screw Fixation in the Lumbar Spine by Using a Cumulative Summation Test. Spine. 2015; 40(2): 87-94. PMid: 00007632-201501150-00005. http://dx.doi.org/10.1097/BRS.0000000000000680

[40] Hinson WH, Kearns WT, Ellis TL, et al. Reducing set-up uncertainty in the elekta stereotactic body frame using stealthstation software. Technology in cancer research \& treatment. 2007; 6(3): 181-6. PMid: ISI:000247665500006. 
[41] Zhang Q, Hu YC, Liu F, et al. Correction of motion artifacts in cone-beam CT using a patient-specific respiratory motion model. Medical physics. 2010; 37(6): 2901-9. PMid:20632601. http://dx.doi.org/10.1118/1.3397460

[42] Rahni AAA, Lewis E, Guy MJ, et al. A Particle Filter Approach to Respiratory Motion Estimation in Nuclear Medicine Imaging. Nuclear Science, IEEE Transactions on. 2011; 58(5): 2276-85. http://dx.doi.org/10.1109/TNS.2011.2162158

[43] King AP, Tsoumpas C, Buerger C, et al., editors. Real-time respiratory motion correction for simultaneous PET-MR using an MR-derived motion model. Nuclear Science Symposium and Medical Imaging Conference (NSS/MIC), 2011 IEEE; $201123-29$ Oct. 2011. http://dx.doi.org/10.1109/nssmic.2011.6153674

[44] Geneser SE, Hinkle JD, Kirby RM, et al. Quantifying variability in radiation dose due to respiratory-induced tumor motion. Medical Image Analysis. 2011; 15(4): 640-9. http://dx.doi.org/10.1016/j.media.2010.07.003.

[45] Fayad H, Pan T, Roux C, et al., editors. A generic respiratory motion model for motion correction in PET/CT. Nuclear Science Symposium Conference Record (NSS/MIC), 2010 IEEE; 2010 Oct. 30 2010-Nov. 62010. http://dx.doi.org/10.1109/nssmic.2010.5874229

[46] McClelland JR, Hawkes DJ, Schaeffter T, et al. Respiratory motion models: a review. Med Image Anal. 2013; 17(1): 19-42. PMid: 23123330. http://dx.doi.org/10.1016/j.media.2012.09.005

[47] Freschi C, Ferrari V, Porcelli F, et al., editors. An Augmented Reality Navigation Guidance for High Intensity Focused Ultrasound Treatment. Conf Proc ICABB, International Conference on Applied Bionics and Biomechanics 2010; 2010; Venice, Italy.

[48] Hacihaliloglu I, Rasoulian A, Rohling RN, et al. Local phase tensor features for 3-D ultrasound to statistical shape+pose spine model registration. IEEE transactions on medical imaging. 2014; 33(11): 2167-79. Epub 2014/07/06. PMid: 24988590. http://dx.doi.org/10.1109/TMI.2014.2332571 